\title{
Algorithm Study and Software Design of District Grid On-Line Risk Assessment Based on Fuzzy Theory
}

\author{
Jing Li ${ }^{1}$, Yadi Luo ${ }^{1}$, Lijie Chen ${ }^{1}$, Donghong Zhao ${ }^{2}$ \\ ${ }^{1}$ China Electric Power Research Institute, Beijing, China \\ ${ }^{2}$ China Wuzhou Engineering Group Co., Ltd., Beijing, China \\ Email: lijing2010@epri.sgcc.com.cn,wuyuanzdh@126.com
}

Received March, 2013

\begin{abstract}
In the background of the design and construction of Smart Grid Operation Supporting System for District Power Networks, this paper established the weighted fault probability model of the overhead line which is based on equipment operating status, utility theory and fuzzy theory. In this model, the proper membership function is adopted to describe the influence of lightning, wind speed, line ice and temperature, and the outage rate of overhead line, derived from historical statistics, is amended. Based on this model, the power supply risk analysis software is developed to calculate the online risk indicators of district grid, and provide real-time decision support information based on risk theory for scheduling operations personnel.
\end{abstract}

Keywords: Power System; Fault Probability Mode; the Overhead Line; Risk Assessment; Fuzzy Theory

\section{Introduction}

The failure of power system is a collection of possibility and severity. When analyzing power system faults, the traditional EMS only took the most likely contingency list, and also did not quantify the effect. In contrast, online operational risk analysis is more scientific, which can not only reflect possibility of contingency, but also severity of power system faults by establishing risk indices[2]. Regional grids are mostly radial network structure. When faults occur, power grid splitting or loss of load would happen, so it is very essential to study online risk assessment model and algorithm applicable to regional grid, which can assist dispatchers making security decision, guarantee sufficient security margin and make full use of power system transmission capacity. It can be a security barrier for power system safety and stability operation.

This paper aims to design online risk analysis software of power supply for the district grid based on the realtime possibility model of elements' faults, and using the fuzzy theory to deal with the uncertainties factors. Otherwise, it can provide real-time risk-based decision-making information for dispatchers.

\section{Basic Concepts}

\subsection{Definition of Operation Risk Assessment}

The basic definition of operational risk assessment of power system is: giving comprehensive measurement of possibility and severity of uncertainty power system faces to[1]. Risk-based security assessment describes possibility of contingency by probability, and denotes the severity of failure by severity function. Then we can get the quantitative risk indices by integrating the two aspects.

The basic formula for calculating risk indices is:

$$
R_{\text {isk }}\left(X_{f}\right)=\sum_{i} P_{r}\left(E_{i}\right) \times S_{e v}\left(E_{i}, X_{f}\right)
$$

Here: $X_{f}$ is power system's operational status; $E_{i}$ is the ith failure and $P_{r}\left(E_{i}\right)$ is its probability; $S_{e v}\left(E_{i}, X_{f}\right)$ denotes the severity level of Ei under the operational status of $X_{f}, R_{\text {isk }}\left(X_{f}\right)$ is a risk index of the operational status $X_{f}$.

\subsection{Contents of Power System Risk Assessment}

Figure 1 shows main content of domestic and foreign study currently about power system operational risk assessment and relationship between them. Risk-based security assessment can be divided three categories: element-level risk assessment, system-level risk assessment

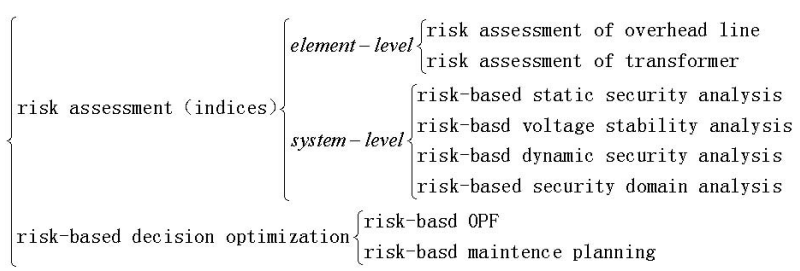

Figure 1. Contents of power system risk assessment. 
and risk-based decision optimization.

The method of power system risk evaluation typically includes 4 steps: determining (1) component outage models; (2) selecting system states and calculating their probabilities; (3) evaluating the consequences of selected system states; (4) calculating the risk indices.

\subsection{Risk Assessment Methods of Power System}

According to different objects' characteristic, different risk assessment methods should be adopted [5]. For simple systems, there are 4 fundamental approaches: the probability convolution, series and parallel networks, Markov equations, and frequency-duration approaches. For a large-scale and complex system, risk assessment methods include status enumeration and Monte Carlo simulation. The latter can be divided into sequential and non-sequential sampling method.

\section{Algorithm Design of Online Power Supply Risk Assessment for Regional Grid}

Regional grid is a composite generation and transmission system, whose risk assessment includes 4 main aspects: determination of component failure and load curve model, selection of system status, identification and analysis system problems, and calculation of reliability indices. Both the status enumeration and Monte Carlo simulation can be applied to regional grid risk evaluation. The two methods use different approaches to select system status and have different forms of formulas to calculate risk indices. The techniques of identifying and analyzing problems in system status are the same. These include power flow calculation and expected contingency analysis for problem recognition and the optimal power flow for remedial actions.

Compared with large-scale grids, regional grid is closedloop design but open-loop operation, and has more complex wiring modes and operation modes. In this paper, we study the grid between $500 \mathrm{kV}$ substation and the load supplied by the substation directly or indirectly. The characteristics are as follows[3]:

1) Complicate voltage level, from $10 \mathrm{kV} / 6 \mathrm{kV}$ to 66 $\mathrm{kV} / 220 \mathrm{kV} / 500 \mathrm{kV}$;

2) Various power source, including large grid and a variety of distributed power source;

3) Coexistence of long and short lines;

4) Coexistence of overhead lines and cable lines;

5) Complex network structure, running in open-loop or weak-link style;

6) Constantly increasing capacity of single load and dynamic load;

7) Part district grid equipments lack some data acquisition or have poor data acquisition such as voltage, reactive power, active power transformer tap because of in- terest, skill level or some special connection, $\mathrm{T}$ tie line, for example.

Online risk assessment is mainly used for dispatching department, only considering steady analysis. Based on present research, the calculation process of online power supply risk analysis for regional grid is illustrated in Figure 2, which doesn't consider the impact of human decision.

\subsection{Status Selection}

Operational risk evaluation for planning department selects system status with state enumeration or Monte Carlo simulation method. The software in this paper is based regional smart grid dispatching technical supporting system, so it can get operation state by fully using telemetry and teleindication data. The probability of present system status is 1 .

\subsection{Network Modeling}

It is not suitable to copy traditional reliability-based "branch-bus model" when modeling power network structure, which is feasible for planning and designing department when approximate model (node model), based on improved state estimation, we obtain network computing model (bus model) by network connectivity analysis, which can be changed with switch state so it can meet the demand of real-time condition.

Regional grid is high voltage distribution network. The fundamental task of regional grid dispatching is to assurance grid's security, economic, high-quality operation,

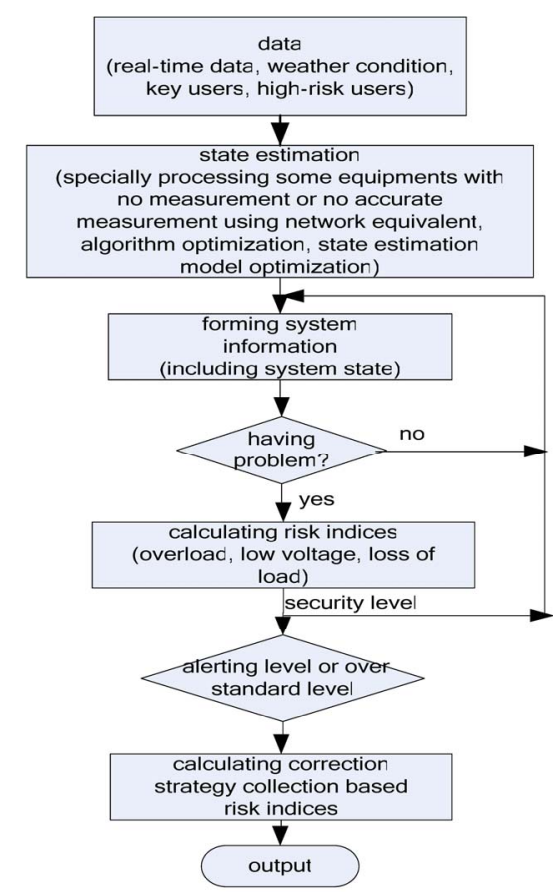

Figure 2. Calculation process of risk indices. 
to guarantee the interests of users, to adapt to the needs of economic construction and people's living, in particular to ensure safety and sustainable power supply for high-risk customers and important users. However, lots of high risk customers and important users connect to grid by low-voltage side, so under current technology, it is difficult to get detailed physical model of the whole grid by SCADA, especially low-voltage side grid. Corresponding to the network which can not model by SCADA, we developed associated model interface of high risk customers and key users by introducing database and visualization technology and making fully use of staff report and statistical data in order to assurance the model of customers in low-voltage side more closely to reality. Associated modeling refers to obtain information of devices being associated by the information of associated devices. Here, associated devices are the devices with telemetry and teleindication data, and the devices being associated are high-risk and important customers.

1) Component Failure Models

The generation unit capacity controlled by regional grid EMS is generally small, so we utilize the two-state (up and down) model as the failure model of generating units, not considering dated states.

Transmission components mainly include overhead lines, cables, transformers, capacitors, and reactors. In general, these components are presented using two-state (up and down) model.

2) Component Real-Time Reliability Model

During the operation of the power system, overhead lines operation conditions are more complex and most severely affected by uncertain factors such as climate environmental and so on, which have different influence characteristics to the overhead lines. In this paper, a method of dealing with uncertainty information based on the fuzzy theory was adopted of appointment, and combined with the actual operation conditions of the power system; the overhead line fault probability model is established. That the failure rate of the overhead lines is the overhead line outage probability multiplied by a correction factor of the weather on the outdoor component outage probability impact. Weather factors affect the rate of overhead line fault considering temperature, wind speed, lightning and Line Icing.

Temperatures, wind speed, lightning and Line Icing is fuzzy uncertain factors, which are different from random factors, there is no exact probability distribution and classical probability statistical methods can not be used to describe it. The fuzzy set theory introduced by Zadeh Professor is a powerful tool to deal with and descript the fuzzy uncertain factors. The fuzzy set allows for the description of concepts in which the boundary is not sharp. Besides, a fuzzy set concerns whether an element be- longs to the set and to what degree it belongs. It does not consider the situations where elements do not belong to. As a result, the range of fuzzy set is in $[0,1]$. A fuzzy set is mathematically defined by Zadeh as:

$$
A=\left\{\left(x, \mu_{A}(x)\right) \mid x \in X\right\}
$$

where is the membership function of in $A$, and $X$ is the universe of objects with elements $\mathrm{x}$. In the case of the classical "crisp" set A, membership of $\mathrm{x}$ in A can be viewed as a characteristic function that can obtain two discrete values:

$$
\mu_{A}(x)= \begin{cases}1 ; & \text { if } x \in A \\ 0 ; & \text { if } x \notin A\end{cases}
$$

For the fuzzy set A, the value of the membership function can be anywhere between 0 and 1, making it different from a crisp set. Membership function of a fuzzy set expresses to what degree the value of $\mathrm{x}$ is compatible with the concept of A.

The method of establishing the membership function include weighted method, fuzzy statistics, expert scoring method, interpolation, standard function method and so on. There is strong uncertainty to the impact of climate change for overhead lines running. In this paper, based on the long-term experience of dispatcher to judge for these types of environmental factors and determine the membership function.

a) The membership function of lightning impact

The density of lightning is an important indicator to determine the lightning degree of a region. Lighting Location System (LLS) can automatically measure and record the density of lightning. Lightning protection design standards also adopt lightning density as a reference. The membership function of the lightning effects identified here as shown in Figure 3:

The membership function of lightning disasters impact on overhead lines running as:

$$
\mu_{1}(x)=\left\{\begin{array}{l}
0, x<a \\
\frac{x-a}{b-a}, a \leq x<b \\
1, x \geq b
\end{array}\right.
$$

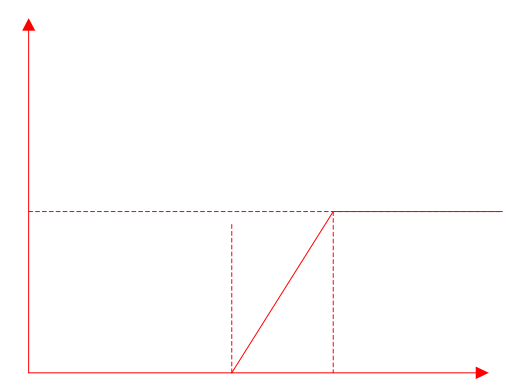

Figure 3. The membership function of the lightning effects. 
where in (4) a and b is the lightning density threshold determined according to the experiences of the dispatching personnel, In other words, it does not affect while the lightning density is less than the lower limit threshold value $a$, and the influence coefficient is 0 , otherwise, higher than the high limit threshold value $b$ is considered a greater impact, influence coefficient is 1 .

b) The membership functions of wind speed and line Icing

Wind speed can be obtained by the meteorological department forecast; while ice thickness for the line, air humidity, temperature and wind size the extent of ice damage has a larger impact, not yet theoretical or empirical model to predict the extent of ice cover based on meteorological conditions, we use the actual ice thickness measurement indicators to assess the severity of the ice storm. Wind speed and line of ice thickness with the fall line health density similar to lighting, the same form of the membership function $\mu 2(x), \mu 3(x)$, shown in Figure 4:

For the wind speed, in $\mu 2(\mathrm{x})$, A is the impact threshold value determined according to the experiences of the dispatching personnel, $\mathrm{b}$ is the critical value determined catastrophe occur; Line Icing $\mu 3(\mathrm{x})$, a and $\mathrm{b}$ are respectively the upper and lower critical value of ice thickness impact.

c) The membership function of temperature impact on overhead lines

The temperature forecast information can be obtained by contact with the meteorological department. Within the normal temperature range, the temperature did not affect the line running, so the value is set at 0 ; when the temperature is too low or too high, its influence is large, and the function value is set to 1 . The membership function shown in Figure 5 below:

The membership functions of temperature impact on overhead lines as:

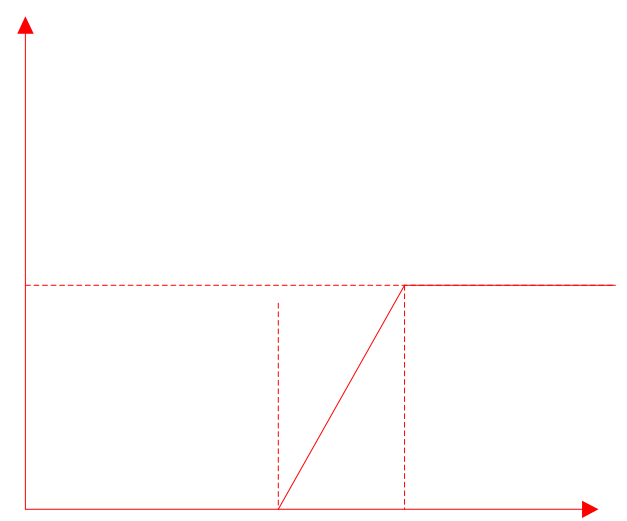

Figure 4. The membership function of the wind speed and line Icing.

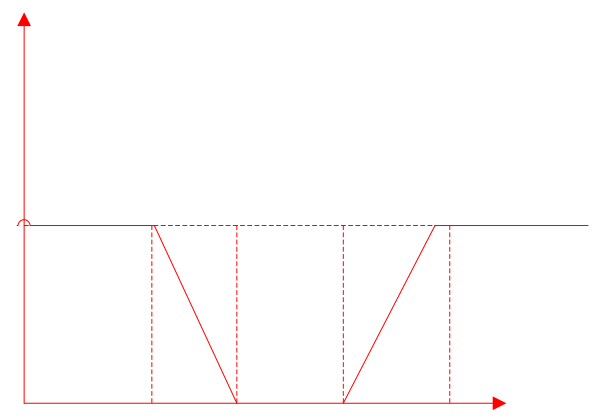

Figure 5. The membership functions of air temperature.

$$
\mu_{4}(x)=\left\{\begin{array}{l}
1, x<c \\
\frac{a-x}{a-c}, c \leq x<a \\
0, a \leq x<b \\
\frac{x-b}{d-b}, b \leq x<d \\
1, x \geq b
\end{array}\right.
$$

where in formula $5, \mathrm{a}, \mathrm{b}, \mathrm{c}$ and d determined according to the experiences of the dispatching personnel are the impact threshold value that air temperature impact on overhead lines running.

Any overhead line segmentation, the fuzzy number vector composed by the membership function of various impact factors is determined as follows

$$
\mathrm{R}=\left[r_{i 1}, r_{i 2}, r_{i 3}, r_{i 4}\right]
$$

where $r_{\mathrm{i} 1}, r_{\mathrm{i} 2}, r_{\mathrm{i} 3}$ and $r_{\mathrm{i} 4}$ successively corresponds the fuzzy numbers that lightning, wind speed, line icing and temperature affect the segment $\mathrm{i}$ line outage probability, Definition B $=[$ B1, B2, B3, B4] as the weight coefficients of line fault outage rate considering four influence factors, then:

$$
\boldsymbol{A}=\boldsymbol{R} \times \boldsymbol{B}=\left[\mu_{1}, \mu_{2}, \cdots, \mu_{n}\right]
$$

where, $\mu_{i}$ is the impact factor of the i-th overhead lines considering the four influential factors. Set the outage rate of overhead lines acquired from historical statistics is $\bar{\lambda}$, and after correction by the influencing factors of overhead line outage rate is $\lambda_{i}$, then:

$$
\lambda_{i}=\left\{\begin{array}{l}
\left(1+\mu_{i}\right) \bar{\lambda},\left(1+\mu_{i}\right) \bar{\lambda}<1 \\
1,\left(1+\mu_{i}\right) \bar{\lambda} \geq 1
\end{array}\right.
$$

\subsection{Selection and Analysis of Expected Fault Collection}

We can obtain the expected fault collection by integrating the following three ways: 1) Scanning the whole grid by $\mathrm{N}-1 ; 2$ ). Scanning the special operation mode with potential power supply risk, such as single line to single 
substation, single power source to substation, then it can be got the fault group which would influence the security of power supply; 3) Defining the fault group by experienced dispatchers and operation analysts via visual manmachine interface;

The expected fault set formatted by 1) and 2) has included most accidents of high frequency and high risk. 3) is only as a necessary complement, which can reduce the manual workload and maintenance.

Online calculation software has high requirements for computing speed. In paper [6], the author combined fault enumeration and probability sampling method, and improved computing speed by parallel computing in the foreground and background. This approach has two shortcomings: first, it increased hardware cost; second, it raised inaccuracy by adopting fault sampling mode. We absorb results of existing "static security analysis" research to analyze fault, which can satisfy computing speed requirement. This fault analyzing method utilized AC-DC hybrid algorithm, and has introduced parallel computing technology based on multi-processor workstation. The approach has been improved by combining with node optimization, matrix inversion and node type conversion, etc, which has greatly improved computing speed. The correctness has been verified by the application in regional-level scheduling, provincial scheduling and city-level scheduling. For the test of 2000 nodes system, it only needs $3 \mathrm{~s}$ scanning whole grid lines, transformers and units. The computing time would slightly increase with the increase of limit violation number.

As important users and high risk customers generally have double power source, if not considering hot backup source in fault analysis, the risk indices of loss of load computed would deviate greatly with actual situation. So when the main source of important users or high risk customers break down, it should be analyzed after putting into backup source.

\subsection{Risk Indices}

It is presented three risk indices appropriate for regional grid in this paper according to formula(1): (1) line overload risk; (2)bus low-voltage risk; (3)loss of load risk.

We compute risk indices (1),(2) by the formula, here: denotes the limit violation of line power flow and bus voltage; X denotes security upper limit or lower limit; the superscript $2 \mathrm{~m}$ is used to overcome the "shelter" defect[4].

The power supply security of important users and high risk customers is related to a range of social, political and economic issues. The outage severity of these users depends not only on the district grid's own characteristic, but also on the users' property. We introduced an importance factor to classify these users, and because of lacking outage time, we only compute loss of load risk indi- ces, not outage cost evaluation indicators. The computing formula of risk (3) is as, here: is the importance of the jth load; denotes the reduction amount of the jth load after failure I; is the load number of reduction.

We adopted real-time failure probability model denoting the probability. For outdoor components, the weather condition value is set by dispatchers; for indoor components, the weather condition value is constantly equal to 0 .

\subsection{Risk Level}

The risk indices can quantify the risk of system, but for dispatchers, it is more expected that the risk indices can directly show the system security condition. So we classified three risk grades according the risk value: security level, alerting level and over standard level.

\subsection{Risk-based Correction Strategy Set}

Distract grid is closed-loop design but open-loop operation. When recovering, the distract high-voltage grid (220 kV and above) and radial distribution grid lower than $110 \mathrm{kV}$, different correction and control measures should be adopted.

a) Correction and Control for High-voltage Distract Grid

Based on sensibility calculation, integrating planning method and objective function selecting, then giving the control target and control variable, the correction measure can be obtained for load and generation unit.

b) Distract Low-voltage Radial Grid Correction Control

For distract grid, the operation mode is usually radial operation under close-loop. It is generally adopted adjusting operation mode as effective measure to ensure continuous power supply and eliminate limit violation. The correction strategy includes load balance, single power source switch, multi-source load transfer, etc. In extreme cases, it can be adjusted by removing load according to load importance. After eliminating fault, system recovery takes into account recovery path constraints and risk indices constraints.

\section{Conclusions}

It is often difficult to determine the possibility of component failure due to lack of statistical data. And the possibility of outdoor component failure has closely relationship with climatic conditions. For power dispatching, it is originally difficult to get weather forecast parameters. Even if it can be obtained the weather forecast parameters, it has possibly an error. In this paper, based on utility theory and probability theory, we have established the three-dimensional model of failure probability by making fully use of the dispatchers' operation experience and 
sensitivity to the weather. After getting the model, when analyzing, it adopt AC-DC hybrid algorithm, and has introduced some advanced technology, including parallel computing, dynamic node ordering optimization, matrix inverse optimization, etc. The expected contingency collection almost includes all accidents of high frequency and high risk. And the computing speed can fully meet the requirement of online analysis of power system. For the characteristic of regional grid, by integrating SCADA, man-machine visual technology, it can assist dispatchers make decisions. It can raise power grid dispatching level from current artificial analytical mode up to automatic smart mode.

\section{REFERENCES}

[1] CIGRE Task Force 38.03.12. Power system security assessment. Electra, Vol. 175, 1997, pp. 49-77.
[2] Wanh, J. D. Mccalley and V. Vittal, "Increasing Thermal Rating By Risk Analysis," IEEE Trans on Power Systems, Vol. 14, No. 3, 1999, pp. 815-828.doi:10.1109/59.780891

[3] W-H. Fu, Mccalleyjd and V. Vittal, "Risk Assessment for Transformer Loading," IEEE Transaction on Power Systems, Vol. 16, No. 3, 2001, pp. 346-353. doi:10.1109/59.932267

[4] Y. Q. Feng, W. C. Wu, H. B. Sun, et a1., "A Preliminary Investigation on Power System Operation Risk Evaluation in the Modern Energy Control Center," Proceedings of the CSEE, Vol. 25, No. 13, 2005, pp. 73-79.

[5] N. Ming, J. D. McCalley, V. Vittal, et a1., Online Risk-based Security Assessment," IEEE Transaction on Power Systems, Vol. 18, No. 2003, pp. 258-265. doi:10.1109/TPWRS.2002.807091

[6] H. J. Shi, G. Fei and M. Ding, "Research on On-line Assessment of Transmission Network Operation Risk," Power System Technology, Vol. 29, No. 6, 2005, pp. 43-48. 